\title{
Estimation of Specimen Lensing in Atom Probe Tomography
}

\author{
D. Haley, G.D.W Smith, C.R.M. Grovenor \\ Department of Materials, Oxford University, Oxford OX1 3PH, United Kingdom
}

Atom probe tomography has been extensively utilised in materials research for decades, with technological advancements rapidly increasing the rate of data acquisition. As a technique, atom probe is uniquely positioned to provide both structural and chemical information at the atomic scale, due to the large magnifications coupled with isotopic mass resolution [1]. Datasets provide rich sources of information due to the combined spatial and chemical information at near-atomic magnifications. However, as data rates have increased, preserving the quality of atom probe information has become increasingly critical. One of the major limitations of the technique is that unlike other common microscopy methods (e.g. SEM, TEM), the lens is not formed in a controlled manner, as the evaporating specimen itself $i$ s the lens [2]. As the tip geometry is continually altered by evaporation, the lensing function constantly changes in an uncontrolled fashion, making plausible reconstruction difficult.

The reconstruction problem is complex; it involves the inversion of the mapping of an unknown projection geometry from external observations and tracking the change through time, as there currently exists no direct observation of the specimen. This renders the quantitative inversion of the specimen's lensing behaviour difficult. The inability to characterise the lens results in large spatial distortions in the positioning of atoms at a virtual specimen surface when using smooth analytical point projection based methods - the modern norm. This work attempts to address the problem in two ways, firstly we develop methods to simulate ion trajectories in APT data from TEM images of APT tips, thus characterising the lens function at any given time, and secondly we apply existing techniques for front tracking in order to observe the time-domain component (surface evolution) of the reconstruction problem.

Figure 1 shows the potential field around a measured aluminium tip, after field evaporation, as computed from TEM observations of the APT tip. We outline procedures for the inversion of the projection function therein, using particle tracking techniques to produce detector to tip mappings through surface estimation and finite element methods.

Secondly, as shown in Figure 2, we show methods based upon the technique of "level sets" [3] for the extrapolation of observed (or proposed) geometries to perform surface front tracking by curvature flow; in the figure the front is eroded at a speed that is proportional to curvature. Subsequently, this front tracking method can accommodate variable rate evaporation, such as is common in multiphase systems.

[1] Kelly, F.; Larson, et al., Atom Probe Tomography of Electronic Materials Annual Reviews of Material Science, (2007), 37, 681-727

[2] Smith, R. \& Walls, J. M., Ion trajectories in the field-ion microscope, Journal of Applied Physics, D, (1978), 11, 409-419 
[3] Sethian, J. Level Set Methods and Fast Marching Methods: Evolving Interfaces in Computational Geometry Fluid Mechanics, Computer Vision and Materials Science.Cambridge University Press, 1999

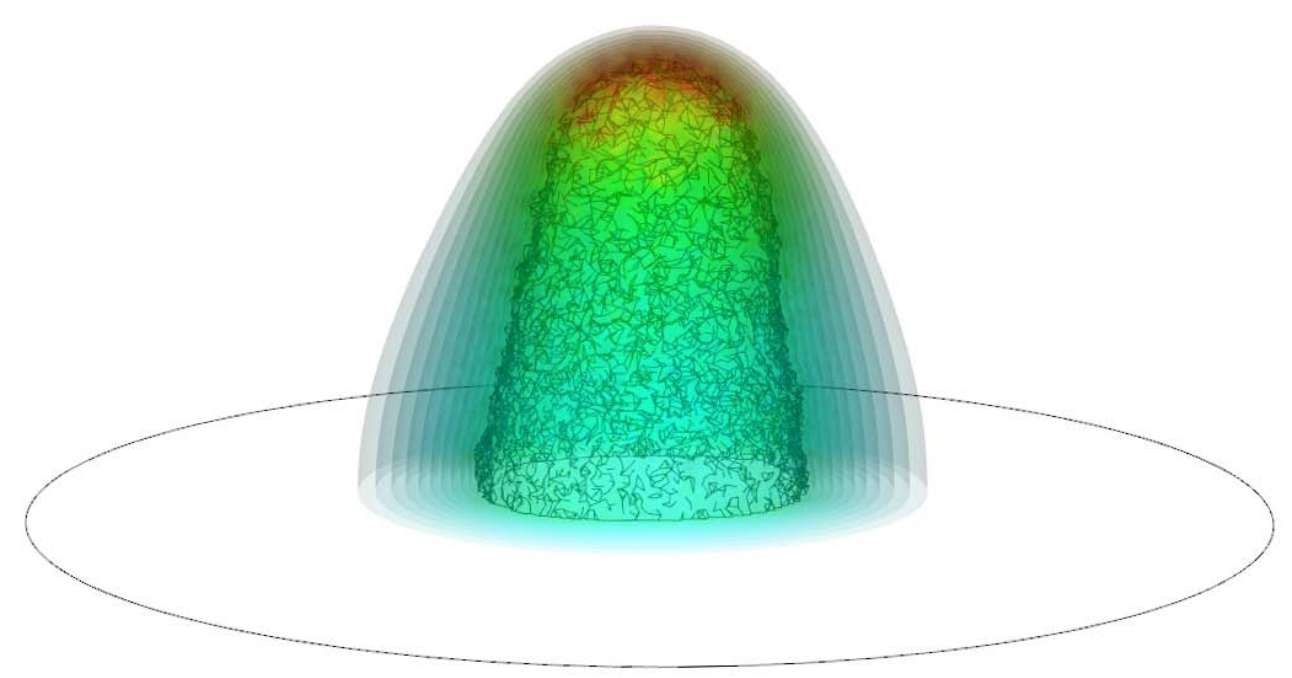

Figure 1: Electric field distribution around aluminium tip, using computed $3 D$ geometery from TEM observations.

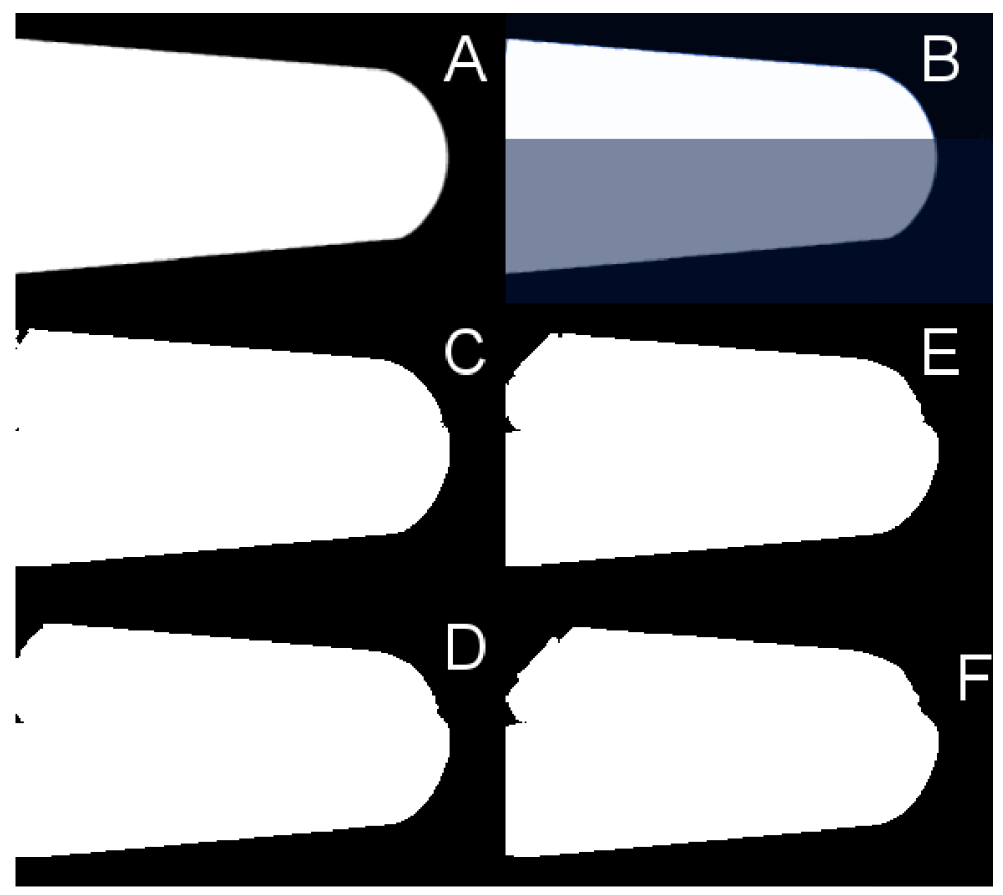

Figure 2: a) Initial synthetic tip shape. b) Speed function for the tip - the lower half of each image is masked with a $1 / 10^{\text {th }}$ speed as compared to the upper half. c-f) Evaporated tip after $2 k, 4 k, 6 k$ and $8 k$ iterations (curvature flow). Left hand evaporation is due to numerical concerns at the image boundary, but does not affect the right hand side. 